\title{
REPRESENTACIÓN DE CONOCIMIENTO: LAS LÓGICAS DESCRIPTIVAS EN UN ENFOQUE ORIENTADO A OBJETOS
}

David Gregorio Maurello Rincón Estudiante de Ingeniería de Sistemas $X$ Semestre Universidad Francisco de Paula Santander

\section{RESUMEN}

La representación de conocimiento es una de las actividades más atra-yentes de la Inteligencia Artificial (Ar-tificial Intelligence: Al), para poder abstraer el saber es necesario tener en cuenta aspectos importantes como el conocimiento de la estruc-tura del lenguaje en que se especifi-ca y el proceso de análisis que se debe aplicar a este lenguaje, las Ló-gicas descriptivas (Description Logics DLs) en el enfoque orientado a objetos nos permiten obtener un formalismo óptimo para la representación, tomando los conceptos del mundo real en que se apoya nuestro conocimiento y las relaciones (roles) entre conceptos, todo esto ayudado con un conjunto de constructores lógicos para completar una

expresividad adecuada a los requerimientos del que expresa.

\section{INTRODUCCION}

Siempre se ha buscado encontrar un soporte a la representación de conocimiento aplicables a diversos campos del lenguaje humano (Human Language: $H L$ ), con el fin de poder definir una expresividad que identifique el saber y del cual se pue-da extraer una arquitectura propia del conocimiento. Se sabe que el conocimiento es primordial e importante para el tratado inteligente de acciones, y su representación es una de las máximas prioridades de la AI. El conocimiento puede ser represen-tado como imágenes mentales en el pensamiento, como palabras habladas o escritas en cualquier lenguaje, en forma gráfica, como cadena de caracteres o colecciones de pulsos eléctricos o magnéticos dentro de un computador.

Las DLs proporcionan un nuevo fundamento a la representación de conocimiento (Knowledge Representation: KR), la semántica y sintaxis que ofrece presenta métodos de efectividad $y$ eficiencia en la formalización del saber que permiten modelar situaciones del mundo real con una arquitectura flexible a la necesidad del que expresa su conocimiento. Las DLs son la base de los lenguajes de conceptos, y estos a su vez entregan un completo marco notacional que permite represen- tar la concepción del saber. Gra- cias a la riqueza semántica y sintáctica que manejan las DLs permite que se posea un enfoque que apoye al desarrollo óptimo de implementaciones del mismo, este enfoque que encaja y logra tener un manejo adecuado de la arquitectura de los Lenguajes de Conceptos es el Orientado a Objetos; este posee como objetivo básico el de obtener un modelo preciso, conciso, comprensible y correcto del entorno donde se desenvuelve la representación de conocimiento, a través de tales aspectos se pretende comprender la expresividad del mismo lenguaje representacional, obteniendo como resultado la especificación de un modelo formal y riguroso de lo que expresan los Lenguajes de Conceptos y permitiendo un grado de abstracción del mundo real que encaja dentro de modelo de objetos.

\section{REPRESENTACION DE CONOCIMIENTO}

Lo que se pretende con el estudio sobre representación del conocimiento en general (no sólo lingüístico) es alcanzar un conocimiento adecuado del cuadro general en el que trabaja la expresividad del HL, y que en muchos estudios lingüísticos es totalmente ignorado.

Tomar el enfoque de Representación = Gramática [3], desafía uno de los requisitos iniciales del representación de conocimiento: la independencia de los datos y la teoría gramatical; puesto que para un contexto general de una representación de conocimiento limita el campo del conocimiento a dicha gramática.

Para abstraer una expresividad propia, que identifique al dominio, se debe encontrar una estandarización en cuanto a arquitecturas sintácticas que representen al conocimiento. Cada implementación del saber tiene su propia arquitectura.

Para poder representar conocimiento se deben tener ciertas elementos primordiales que permitirán expresar y substraer en forma coherente y caracterizar los conceptos y las relaciones entre estos. Identificado en el dominio el conocimiento y el proceso de análisis.

\section{El Conocimiento}

Para poder representar un conocimiento de un dominio se debe poseer un conocimiento previo sobre la contextura del mismo, en térmi- 
nos generales, se pueden identificar al menos cinco tipos de conocimiento que son relevantes en cualquier sistema que procese el lenguaje en el que el dominio se encuentre, estos son:

1. Conocimiento fonológico: Es la información sobre el sistema de sonidos y la estructura de las palabras y las expresiones, los patrones de acentuación, la entonación, etc.

2. Conocimiento Morfológico: Es la información sobre la estructura de las palabras; por ejemplo: el morfema ' $s$ ' se añade a los nombres para formar el plural.

3. Conocimiento Sintáctico: Es la información sobre la organización de las palabras en frases y oraciones; por ejemplo, el cambio en el orden de las palabras para formar oraciones interrogativas.

4. Conocimiento Semántico: Es la información sobre el significado de las palabras y de cómo esos significados se combinan para formar el significado de las oraciones; por ejemplo, el concepto denotado por pegar implica dos entidades: un agente (el que pega) y un paciente (la persona o cosa a la que el agente pega) y esas entidades estarán representadas, correspondientemente, en el sujeto y en el objeto del verbo.

5. Conocimiento Pragmático: Información que puede ser considerada como central en muchas tareas específicas que sistemas de procesamiento de lenguaje del dominio han de realizar, por ejemplo, la recuperación de los referentes de los pro- nombres 0 de las oraciones elípticas, el análisis de las presuposiciones del hablante o de las intenciones comunicativas que subyacen en una frase en particular. Casi todas estas tareas implican información sobre la intención y la disposición de los interlocutores sobre el contexto extralingüístico, etc.

Estos tipos de conocimiento generalmente están especificados como reglas dentro de algoritmos que procesan dicho dominio, para así substraer el enfoque lógico del conocimiento.

\section{El Proceso de Análisis}

El proceso de análisis típico es el que se desprende naturalmente de los distintos niveles de conocimiento. Este proceso es aplicable a muchos campos que intenten procesar conocimientos de un dominio.

1. Análisis Morfológico: Se analizan las cadenas de entrada y se les asigna una o varios lemas (lematización). En este proceso se evalúan los signos de puntuación y o bien se les asigna una función determinada o son descartados.

2. Análisis Sintáctico: Se transforman las secuencias lineales de palabras en estructuras que muestran la forma en que las palabras se relacionan entre sí.

3. Análisis Semántico: Se asignan significados a las estructuras generadas por el analizador sintáctico, es decir se establecen correspondencias entre las estructuras sintácticas y los objetos del dominio.
4. Interacción del discurso: El significado de una frase puede depender de las frases precedentes $y$ también modificar el de las frases siguientes.

5. Análisis Pragmático: La estructura de representación obtenida se reinterpreta para determinar su significado real y puntual dentro del contexto especifico.

Este cuadro ideal que expresa el proceso de análisis no encaja mucho en la realidad, la mayoría de los sistemas que intentan representar conocimiento, no van más allá del análisis sintáctico. La dificultad que entraña el análisis de los niveles posteriores es evidente y además depende en gran medida del éxito obtenido en los análisis precedentes.

En definitiva esta división planteada es valida, no solo para el análisis computacional, sino para el análisis lingüístico.

Cabe recalcar que el procesamiento del lenguaje natural (NLP: Natural Language Processing) y por ende la representación de conocimientos como estudio del mismo, tiene como finalidad la automatización de los procesos lingüísticos, haciendo converger los intereses de lingüistas computacionales, psicolingüistas, informáticos e ingenieros de sistemas; todos ellos desde diferentes perspectivas teóricas y prácticas, intentan desarrollar una teórica que sea totalmente explicita (y por tanto automatizable) de los procesos lingüísticos. 
Actualmente se puede deducir que los problemas más importantes de la representación de conocimiento son:

- La necesidad de un conocimiento profundo de nuestro propio conocimiento. Es evidente que no se posee una comprensión lo suficientemente detallada de muchas materias o áreas de la ciencia. Entre ellas está la que a todos nos concierne, el lenguaje natural. Cuanto más amplio sea nuestro conocimiento del HL, más fácil será recrear sus mecanismos artificialmente. En especial se debería conocer con exactitud tres puntos cruciales:

¿Cuáles son los individuos que constituyen el universo?

¿Qué propiedades poseen? ¿Qué relaciones existen entre ellos?

-El desarrollo de una notación precisa. Aun suponiendo que se posee un conocimiento lo suficientemente detallado de la materia que pretendemos representar, resulta crucial el disponer de una notación lo suficientemente precisa como para poder plasmar ese conocimiento de forma que pueda ser usado de forma inteligente por un cerebro electrónico.

El conocimiento requiere de un esquema de representación el cual se centra en dos aspectos [10]:

-Su capacidad o adecuación expresiva, es decir, lo que el sistema puede «entender» 0 «decir».

Su eficacia notacional.

Estos dos aspectos demuestran la solidez de representación que ofrece el esquema que se emplee, pro- porcionando una óptima modelación del conocimiento.

Un sistema de representación de conocimiento debe ofrecer un conjunto de servicios que permita la jerarquización, la gestión, la modificación y la recuperación de información del conocimiento en cuestión. El sistema debe dar respuestas a cuestiones [8] como:

¿Es un concepto interpretado en algún sentido? (satisfactibilidad).

¿Es un concepto más general que otro? (subsumición)

¿Dónde se ubica un concepto dentro de la jerarquía de conceptos? (clasificación)

¿Es consistente una base de conocimiento? (consistencia)

¿Qué hechos son deducibles de un conocimiento? (instanciación)

¿Cuáles son los conceptos que instancian a un individuo? (comprensión)

¿Cuáles son las instancias de un concepto dado? (recuperación)

Debido a las necesidades propias para la representación adecuada de conocimiento la existencia de lenguajes de representación, facilitan la administración propia de lo que se expresa y proporcionan un modelo concreto y lógico del contenido manejado en la realidad.

\section{LENGUAJES DE REPRESENTA- CIÓN DE CONOCIMIENTO}

Para definir o expresar las frases es necesario un lenguaje de representación del conocimiento, que podría ser el mismo que el utilizado para representar la forma lógica de una frase, aunque normalmente difieren, y esto parece ser el mejor compromiso de diseño, debido a la no existencia de un lenguaje con la suficiente capacidad expresiva para la forma lógica y la suficiente efectividad para la representación del conocimiento.

Aunque la representación abstracta de referencia más utilizada es el cálculo predicador de primer orden, existen distintas soluciones (formalismo) ampliamente utilizadas $y$ aceptadas para representar el conocimiento.

Los distintos formalismos más utilizados para representar conocimiento y para razonar son:

Basado en la lógica (lógica descriptiva)

- Redes semánticas (semántica procedimental)

- Marcos y guiones (Representación estructurada o estereotipo de objetos)

Basado en reglas

- Redes neuronales (Método cone xionista)

- Representaciones híbridas del conocimiento.

Cada uno de estos enfoques representacionales han tratado de ejemplarizar un modelo que se ajusta al del HL, más orientada a su manejo computacional y matemático que a la misma familiarización con las personas que lo necesitan.

Uno de los compromisos siguientes será el de encontrar un modelo que facilite el traslado del conocimiento a dichos formalismos. 


\section{LAS LÓGICAS DESCRIPTIVAS Y LOS LENGUAJES DE CONCEPTOS}

Las DLs forman una familia de formalismos bien determinados, relacionadas con las redes semánticas pero con la característica que la semántica está formalmente definida [10]. Las DLs permiten expresar conocimiento estructurado por medio de expresiones de conceptos y roles.

\section{Los Lenguajes de conceptos :}

-Son un medio de expresar conocimiento taxonómico para describir jerarquías entre conceptos.

Son un lenguaje formal que soporta conceptos y roles (relaciones entre conceptos).

Son un lenguaje permite representar el conocimiento de un dominio por medio de conceptos y roles.

Se conciben como sublenguajes de la lógica de predicados y pueden ser dotados de la semántica declarativa de Tarski.

Abstrayendo de las descripciones anteriores se puede enmarcar dos aspectos importantes en los que trabaja el lenguaje de conceptos, basado en la riqueza semántica de las lógicas descriptivas que son: Los Conceptos y los Roles. Pero los Lenguajes de Conceptos no solo manejan estos dos términos, sino que poseen un conjunto de elementos que enriquecen el KR como son los Constructores Lógicos, estos a su vez apoyan la expresividad con que se representa el conocimiento; estos tres elementos que combinan con una sintaxis y semántica definida para formar las expresiones de conceptos que son las que poseen el formalismo del conocimiento.

Ejemplo de Conceptos: Ejemplo de Roles: Constructores:

$\begin{array}{ll}\text { Hombre } & \text { Hijo } \\ \text { Humano } & \text { Género } \\ \text { Mujer } & \text { Esposo } \\ \text { Persona } & \text { Primo } \\ \text { Graduado } & \text { Amigo }\end{array}$

Negación

$\neg$ Subsumición

Restricción al menos

Restricción a lo más

Conector Disyunción

Conector Conjunción

Igualdad

= Cuantificador Universal?

${ }^{\forall}$ Cuantificador Existencial
El propósito del KR es organizar la información requerida en un formato, para poder acceder a ella y poder tomar decisiones, planificar, reconocer objetos o situaciones, analizar escenarios, llegar a conclusiones o realizar otras funciones cognoscitivas, este formato constituye uno de los aspectos más importantes del KR en el lenguaje de conceptos, porque se ve enmarcado a través de la base de conocimientos, donde se logra especificar en forma detallada la representación del conocimiento.

Una característica general de los sistemas basados en conceptos es que la base de conocimientos consta de: -El conocimiento terminológico llamado TBox

-El conocimiento aseverativo llamada ABox,

El TBox es un esquema general relativo a las clases de individuos a ser representados, sus propiedades generales y mutuas relaciones; mientras que el ABox es una instancia del esquema general TBox, que contiene aseveraciones relativas a individuos, a clases o a relaciones entre los individuos.

Por ejemplo si tenemos la siguiente expresión de conceptos: una Persona que tiene al menos un hijo Graduado, se describe como:

El TBox sería:

Persona $\mid$ ( $\exists$ Hijo.Graduado)

Y el ABox:

Persona $(\mathrm{x}) \wedge(\exists \mathrm{y} . \operatorname{Hijo}(\mathrm{x}, \mathrm{y}) \wedge$ Graduado (y) 
Una base de conocimiento terminológico consta de un número finito de axiomas que corresponden a definiciones o inclusiones de nuevos conceptos.

Una base de conocimiento terminológico o una terminología es un conjunto de axiomas que permiten introducir nuevos conceptos a través de las relaciones de subsumición o de igualdad expresadas por la forma: CN C, o, CN - C respectivamente, donde $\mathrm{CN}$ es un nombre de concepto y $\mathrm{C}$ una expresión de conceptos.

Ejemplo de definición con la siguiente expresión:

Mujer - Humano $\mid\rceil$ Hembra

Este axioma introduce el concepto $\mathrm{Mu}$ jer, afirma que una Mujer es necesariamente un Humano y Hembra y también afirma que un individuo que satisfaga la condición de ser un Huma-no y Hembra es suficiente para inferir que este individuo es una Mujer.

Ejemplo de subsumición de concepto con la siguiente expresión:

\section{Humano Animal Il Bípedo}

Este axioma introduce el concepto de Humano y afirma que un Humano es necesariamente un Animal y un Bípedo pero un individuo que satisfaga la condición de Animal y de Bípedo no implica que sea un Humano.

Los nombres de conceptos que están asociados con la relación de subsumición son conocidos como conceptos primitivos, mientras que aquellos nombres de conceptos que están asociados con la relación de igualdad son conocidos como conceptos definidos. Los conceptos primitivos no están totalmente definidos, ellos tienen sólo características necesarias. Los conceptos definidos están determinados por sus características las cuales son suficientes y necesarias para tener el concepto.

Los nombres de conceptos en una terminología que no son conceptos primitivos, ni conceptos definidos se denominan conceptos atómicos, de ellos sólo se conoce sus nombres y que sus interpretaciones son subconjuntos del dominio de aplicación.

Ejemplo de concepto atómico: Hembra

Con esto tenemos que una DL está caracterizada por un conjunto de nombres de conceptos, un conjunto de nombres de roles y un conjunto de individuos.

Adicional al conocimiento terminológico descrito en TBox, situaciones concretas son descritas en la componente llamada ABox, posiblemente usando los conceptos definidos en TBox. Las situaciones concretas se describen usando nombres de individuos $a, b, \ldots$ que corresponden a elementos de un dominio de interpretación, ejemplo: Juan, es un individuo que se instancia en el concepto Hombre, Así:

\section{Hombre (Juan)}

La componente extensional ABox de una base de conocimientos contie- ne aseveraciones de individuos sobre los conceptos y los roles. Dada una terminología $\mathrm{T}$, una ABox-aseveración tiene una de las siguientes formas:

a : C aseveración de la pertenencia del individuo a al concepto $\mathrm{C}$.

a $\mathrm{R} b$ aseveración de la pertenencia de los individuos a y $b$ al rol $R$.

Con los párrafos anteriores se puede deducir:

-Los lenguajes de conceptos poseen tres aspectos importantes para el KR que son:

- Conceptos

- Roles

- Constructores.

-Para la representación de conocimiento en los lenguajes de conceptos es necesario una base de conocimiento que apoye la administración de los conceptos, especificada esta en dos aséctos básicos:

- El TBox

- El ABox

-Los lenguajes de conceptos tienen como base de todo conocimiento:

- La expresión de conceptos.

-Un concepto puede ser:

- Primitivo

- Definido

- Atómico.

-Las DLs se caracterizan por:

- Nombre de Conceptos.

- Nombre de Roles.

- Nombres de Individuos.

Para continuar es necesario expresar el desarrollo expresivo que ma- 
nejan los lenguajes de conceptos y lo hacen a través de un Lenguaje de Atributos de Conceptos ALC [13], donde definen las expresiones de conceptos de la siguiente manera:

$$
\left.\mathrm{CN}|\mathrm{T}|^{\perp}|\neg \mathrm{C}| \mathrm{C}|\mathrm{D}| \mathrm{C} \mid\right\rfloor_{\mathrm{D}} \mid \leftrightarrow \text { R.C } \mid \exists \text { R.C }
$$

\section{Donde:}

.CN: Es un nombre de concepto.

.C y D: Son expresiones de conceptos. $\cdot R$ :

es un nombre de rol.

- Los nombres de conceptos T (top) y $\perp$ (bottom): representan el concepto más general y el concepto menos general respectivamente.
EL ALCN Anexa dos expresiones más para darle más riqueza a la expresividad de ALC, al permitir conceptos con restricciones de número, expresiones de la forma $(£ n R)$ y $\left({ }^{3} \mathrm{~m} \mathrm{R}\right)$ donde $n$ y $\mathrm{m}$ son enteros no negativos y $R$ es un nombre de un rol [Hollunder y Nutt 1990 ].

En [Hollunder y Baader 1991] se presenta una variante más expresiva de las restricciones de número, llamada restricción de número modificada cuya presentación es de la forma ( $\geq n$ R.C ) o ( $\leq n$ R.C ).

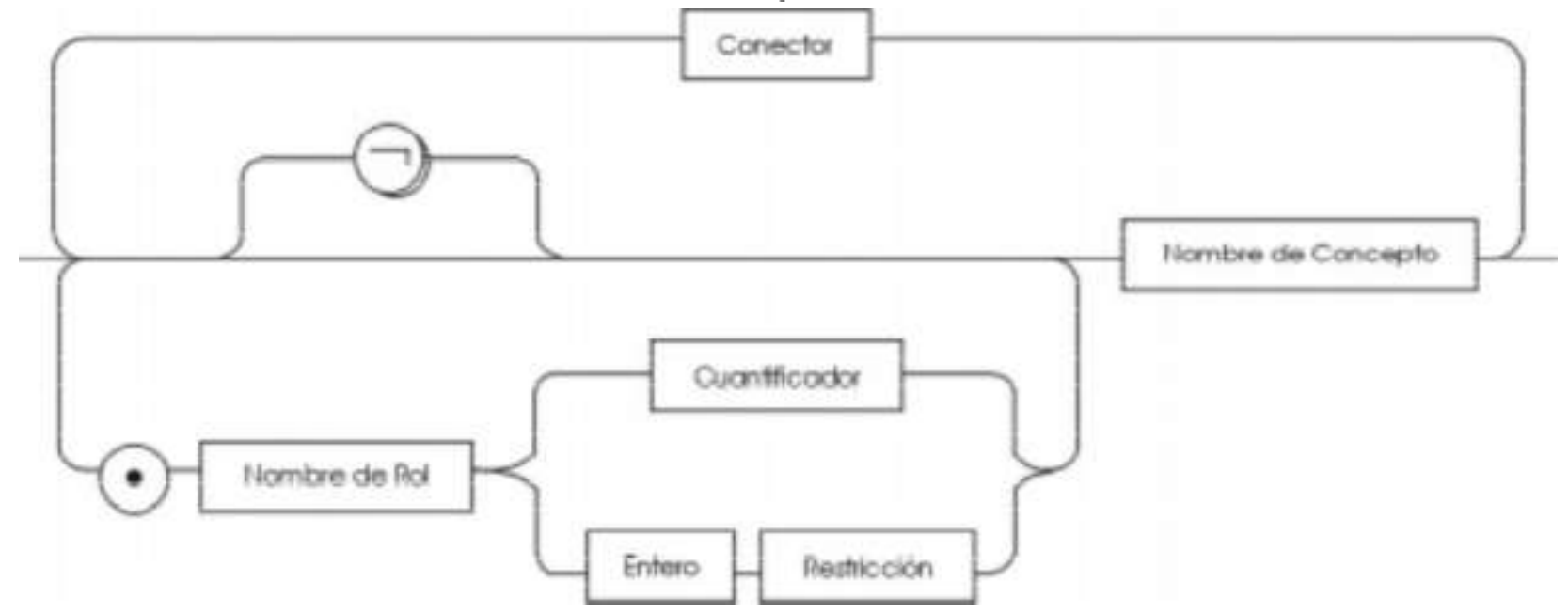

Diagrama BNF de la sintaxis del ALCN enriquecido

\section{LOS LENGUAJES DE CONCEPTOS EN UN ENFOQUE ORIENTADO A OBJETOS}

La posibilidad de crear un modelo formal que me permita manejar el potencial que ofrecen la lógicas descriptivas en sistemas de representación y gestión de conocimiento, fundamentado en técnicas de administración del conocimiento, permitirá adaptar un modelo computacional que facilite y optimice la comprensión del modelo real de la representación del saber. Los Lenguajes de Conceptos logran implantarse con facilidad en un modelo de objetos, dichas características mane-jadas en esta hacen un empalme eficiente de lo que se desea lograr.

Los lenguajes de conceptos permiten representar el conocimiento de un dominio por medio de conceptos y roles, donde los conceptos modelan clases de individuos y los roles especifican relaciones entre las clases. De la combinación de conceptos atómicos y roles atómicos (los cuales están descritos únicamente con su nombre) con adecuados constructores se generan expresiones de conceptos. Los conceptos y los roles son interpretados mediante la semántica declarativa al estilo de Tarski que permite concebirlos como conjuntos y relaciones binarias respectivamente. De esta forma los lenguajes de conceptos son sublenguajes de la lógica de predicados.

Con el análisis realizado se presenta un lenguaje de conceptos que ofrece un potencial expresivo para la $K R$, las DLs dan la fortaleza a este planteamiento, puesto que 
ofrecen por medio de una sintaxis y semántica definida una solución a la representación de conocimiento.

La Al siempre se ha preocupado por la sistematización del conocimiento. Para realizar esta tarea se requieren métodos que permitan representar el conocimiento junto con procedimientos efectivos y eficientes para recuperar e inferir conocimiento [Obermeier 1989, Cote er al., 1993 Horrocks, Rector y Goble
1996, Rector y Horrocks 1997, Bullock, y Goble 1998, Goble 1998]. Esta tarea se realiza por medio de los sistemas de representación de conocimiento (KRSs: Knowledge Representation Systems) mediante el uso de los lenguajes terminológicos o lenguajes de conceptos basados en DLs. Uno de los mecanismos básicos de la representación y procesamiento del conocimiento humano es la división del mundo en clases o conceptos los cuales usualmente están dotados de una jerarquía estructural.

Dado lo anterior podemos expresar el siguiente esquema que me permite poseer un contexto estructurado de manejo de los conceptos para la representación y el potencial expresivo que permitirá el modelo de objetos para la representación de la expresiones de conceptos, que son el formato base del conocimiento, y que este permite constituir el TBox.

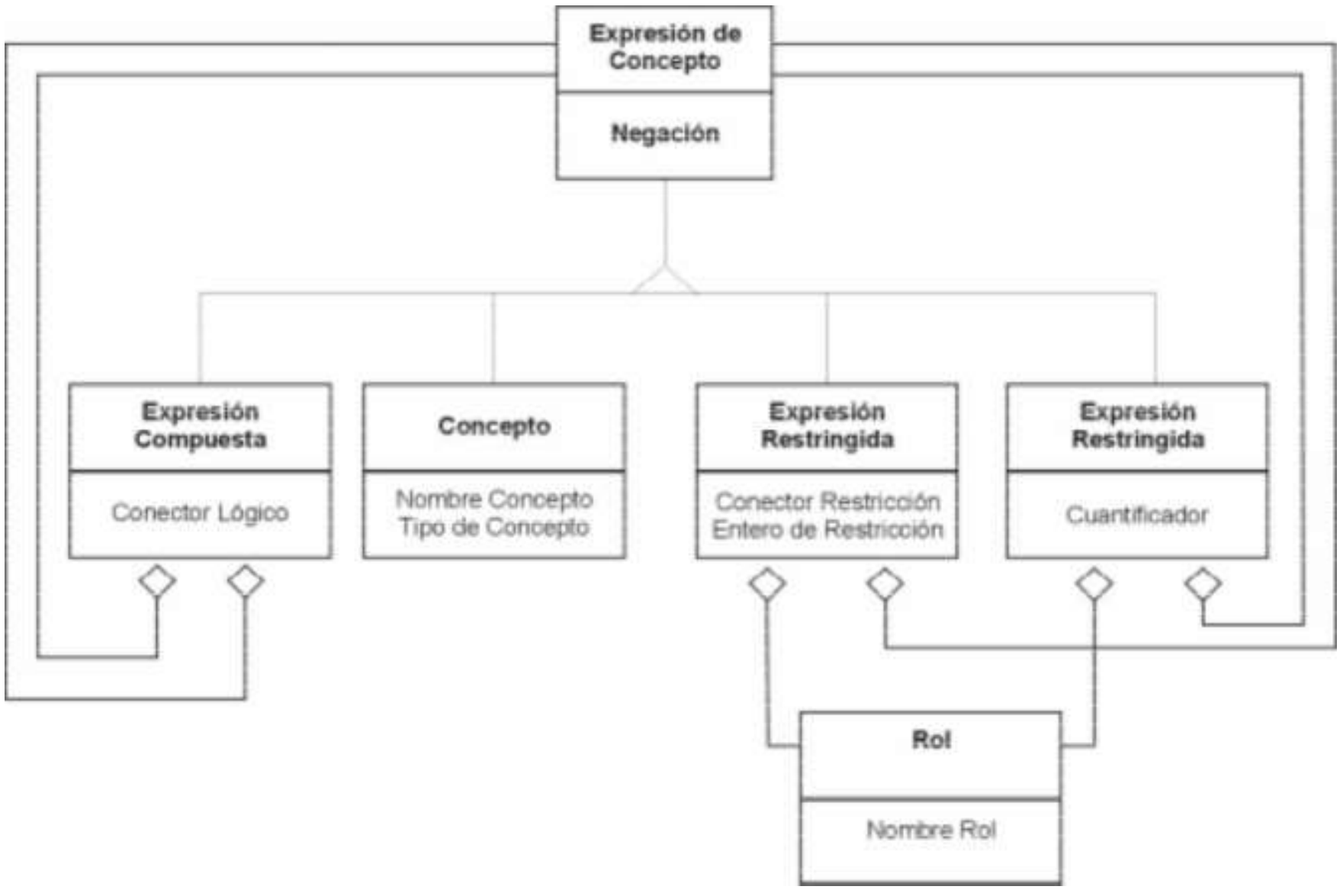


Para la expresión de concepto:

Mujer - Humano 11 Hembra

Podemos visualizar la instanciación siguiente:

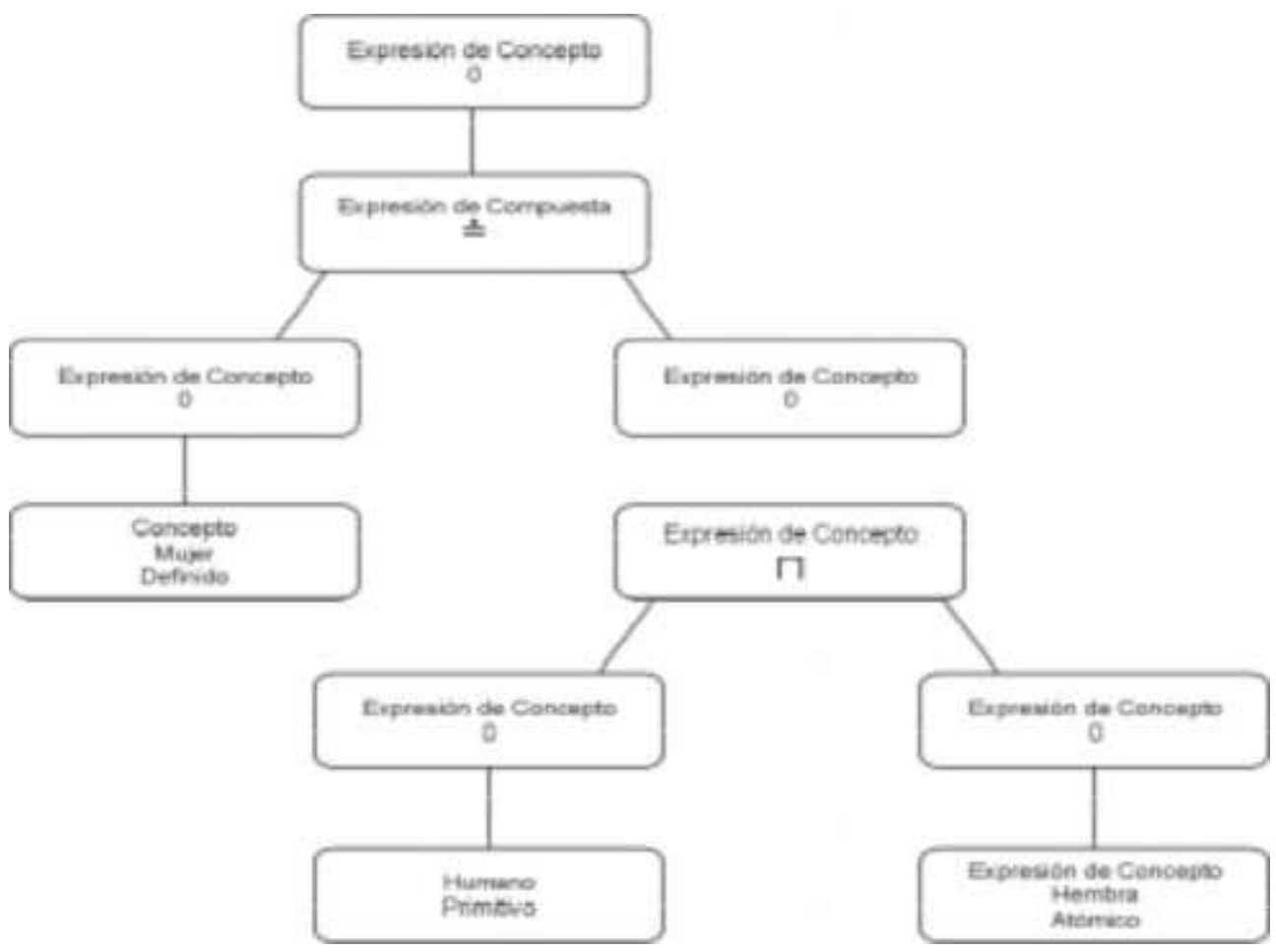

Diagrama de instancia del concepto Mujer

Con esto se observa que el modelo de objetos nos permite obtener una representación de la expresión de concepto que encaja en el mundo real, y que permite administrar en una forma sistematizada el conocimiento.
Sin embargo, se presume que el sistema de representación que maneje el modelo de objetos debe poseer una interacción adecuada entre la persona que posee el conocimiento y el mismo sistema. La representación de expresiones de conceptos por un conocedor de un área o tema estará dada así:

Nombre de concepto,

Nombre de Roles, Conector,

Tipo de concepto, Cuantificador,

Restricción, Entero, Negación, Continuar

\begin{tabular}{|c|c|}
\hline Usuario & \\
\hline
\end{tabular}

Diagrama de flujo de sucesos para la clase Expresión de Concepto. 
Se ha determinado entonces que una clase usuario es necesaria adaptarla al sistema para adecuar mejor el sistema de representación, de la misma forma esto nos permite especificar un diagrama de estados en la clase Expresión de concepto para poder especificar como va ha realizarse el flujo de información dentro del sistema.
Teniendo en cuenta que una expresión que define 0 incluye un concepto va estar dada por va ha estar dada por:

$$
\begin{aligned}
& \mathrm{CN}|\mathrm{T}| \perp \neg \mathrm{C}|\mathrm{C}||\mathrm{D}| \mathrm{C}|| \mathrm{D}|\leftrightarrow \mathrm{R} . \mathrm{C}| \exists \\
& \text { R.C } \mid \geq \text { n R.C } \mid \leq \mathrm{n} \text { R.C }
\end{aligned}
$$

Se puede especificar lo siguiente:

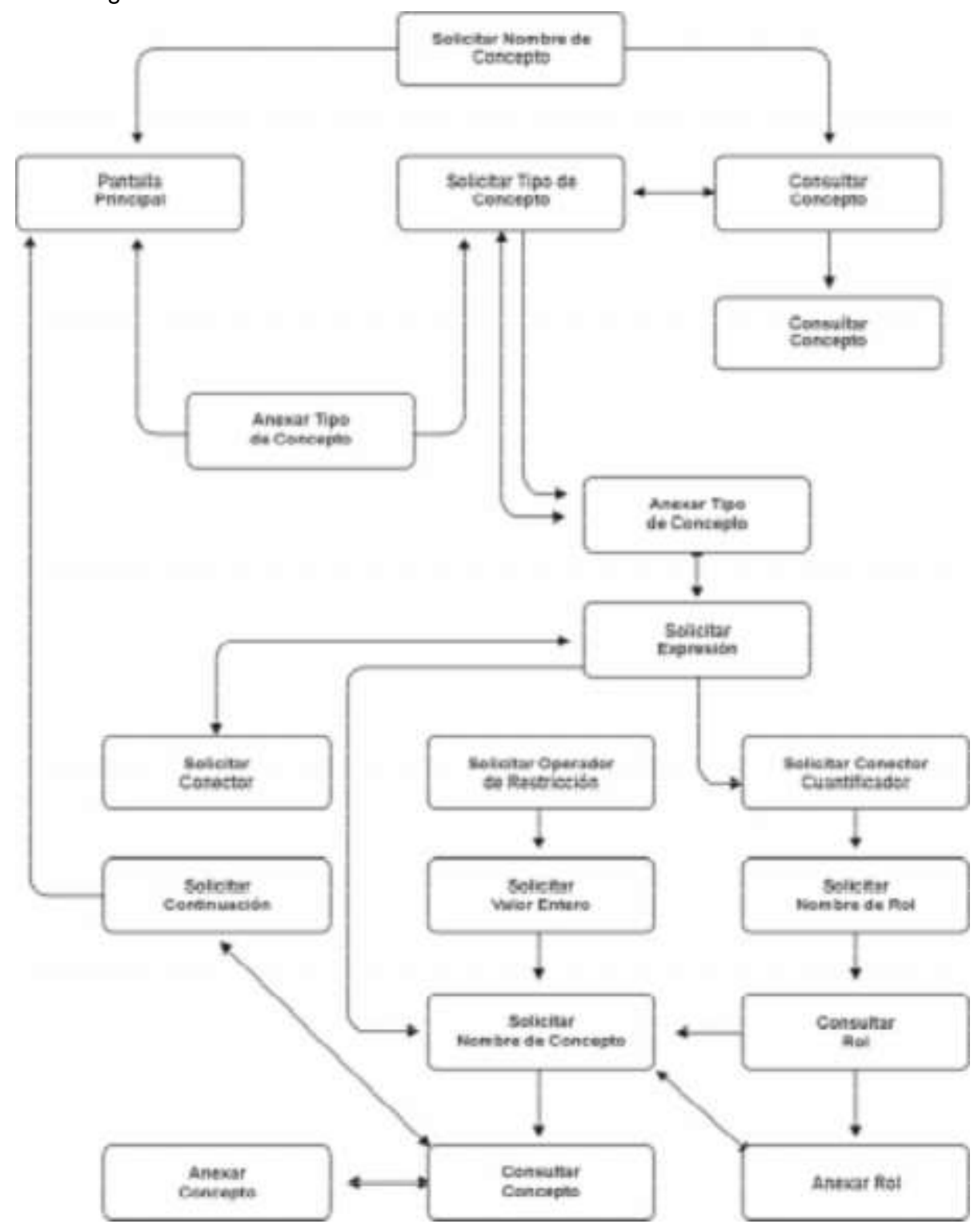

\section{Diagrama de estados para la Clase Expresión de Conceptos}

Con lo anterior se pretende dar un formalismo a la representación de conocimiento con el fin de poder administrarlo adecuadamente y que la persona que lo repre- sente tenga un parámetro fácil de poder especificar las cosas del mundo real que domina sin mecanismos de codificación complicados de entender. 


\section{CONCLUSIONES Y TRABAJOS FUTUROS}

Lo anterior permite mostrar un enfoque que facilita la representación del conocimiento con un potencial expresivo adecuado a muchas áreas del conocimiento, dando pie al almacenamiento jerarquizado $y$ clasificado de las abstracciones de conceptos manejados en el entor- no real de las cosas. Estás abstracciones representadas pueden ser modeladas bajo el enfoque orientado a objetos, aprovechando las características que esto ofrece como son la herencia, encapsulado y estructuración adecuada de la información.
BULLOCK J., and GOBLE C., TouirsT. The Application of a Descrip-tion Logic based Semantic Hypermedia System for Tourism. In Proceddings of Hypertext'98. Pitts-burgh. 1998.

COTE R., ROTHWELL D., PALOTAY J., BECKET R., and BROCHU L. The Systemised Nomenclature of Medicine : SNOMED International. College of American Pathologists. Northfield. 1993.

GAZDAR, G. \& C. MELLISH Natural Language Processing in Prolog. Cambridge. Mass: Addison-Wesley Publishing Company. 1989.

GOBLE C., PATON N., BAKER P., BRASS A., BECHHOFER S. and STEVENS R. Transparent Access to Multiple Biological Information Sources. Journal Intelligence Information Systems. 1998.

HOLLUNDER B and NUTT W. Subsumption Algorithms for Concept Languages DFKI report. RR-90-04. 1990.

\section{BIBLIOGRAFÍA}

HOLLUNDER B. and BAADER F. Qualifying number restrictions in concept languages. In Proceedings of the Second International Conference on the Principles of Knowledge Representation and Reasoning (KR91) Boston. 1991. p. 335-346.

HORROCKS I., Rector A., and GOBLE C. A description logic based schema for the classification of medical data. In Baader et al., 1996. p. $24-28$

LIZCANO BUENO., Luis Ignacio. y OJEDA, Luis R. Una Propuesta de la Lógica Terminológica: El Lenguaje de Conceptos. Reporte Técnico. UFPSUNAL. 2001.

MAC GREGOR R., The evolving technology of classification-based knowledge representation systems, Principles of semantic networks: Explorations in the representation knowledge. Morgan-Kaufman. San Mateo. 1991.

NOWLAN W., Rector A., KAY S., HORAN B. and Wilson A. A Patient
Quedan metas a desarrollarse como la ampliación de la expresividad y el procesamiento del lenguaje natural para poder extraer las expresiones que definan o incluyan conceptos.
Care Workstation Based on User Centred Design and a Formal Theory of Medical Terminology : PEN\&PAD and the SMK Formalism. In Fifteenth Annual Symposium on Computer Applications in Medical Care. Proceedings of SCAM91. 1991. p. 855857

OBERMEIER, K. K. Natural Language Processing Technologies in Artificial Intelligence. Chichester, West Sussex: Ellis Horwood Limited. 1989.

RECTOR A., and HORROCKS I. Experience building a large, reusable medical ontology using a descrip-tion logic with transitivity and con-cepts inclusions. In Proceedings of the Workshop on Ontological Engineering, AAAI, Menlo Park, California, 1997.

\section{SCHMIDT-SCHAUb M. and SMOLKA}

G. Attributive concept descriptions with union and complements. Artificial In-telligence. 1991. $48 \mathrm{p}$. 
